doi:10.17659/01.2019.0036

Journal of Case Reports 2019;9(3):139-141

\title{
Symmetrical Synchronous Stress Fractures of Bilateral Tibial Medial Condyles
}

\author{
Manjot Kaur, Naresh Kumar Garg \\ Department of Radiodiagnosis, Adesh Institute of Medical Sciences and Research, Bathinda, Punjab, India.
}

\section{Corresponding Author: \\ Dr Manjot Kaur \\ Email:drmanjot75@gmail.com}

This is an Open Access article distributed under the terms of the Creative Commons Attribution License (creativecommons.org/ licenses/by/3.0).

Received : April 20,2019

Accepted : June 15,2019

Published : July 5, 2019

\begin{abstract}
Background: Stress fractures are microscopic fractures caused by repetitive cumulative micro trauma on the bone over a period of time. Stress fracture is seen to occur in military personnel and sports persons who indulge in intense physical activity. It causes morbidity, economic loss and long periods of absence from duty and training. Tibia stress fractures are more commonly unilateral and are usually in the diaphyseal portion of the tibia. Case Report: We present a case of bilateral symmetrical synchronous stress fractures of medial condyles of bilateral tibia in a military man. X-ray, computed tomography and MRI of the knees revealed the stress fractures. A symmetrical and synchronous presence of stress fractures in the lower limbs is unusual. Conclusion: Radiological investigations are conclusive in cases of stress fractures. In case of unilateral stress fracture, contralateral side should also be investigated to diagnose bilateral injury.
\end{abstract}

Keywords: Computed Tomography (CT), Fractures, Magnetic Resonance Imaging, Stress Fractures, Tibia.

\section{Introduction}

Stress fracture is an overuse injury. The term stress fracture is used to describe a fracture that occurs in a normal bone after repetitive stress, none of which is individually capable of causing a fracture. During intense exercise, bone resorption exceeds bone formation although the exact mechanism remains unclear [1]. Patients usually present with worsening pain with a history of minimal or no trauma. In the weight bearing lower limb, there is often a history of a recent increase of physical activity or significant alteration in the type or duration of normal athletic activity. Athletes and military men are subjected to change in types of training and training intensities and thus are at increased risk of developing a stress fracture. The most common sites of stress fractures are femoral neck, anterior tibial cortex, metatarsals, talar neck. A discomfort during physical activity can be the initial manifestation, evolving to constant pain at rest.

\section{Case Report}

A 40 year-old military male was referred to our radiology department with a two month history of atraumatic bilateral knee pain. He reported knee pain after first week of vigorous exercise consisting of jumping and running. As the intensity of pain was more on right side, he was referred for MRI of the right knee joint. On examination, he had tenderness over the medial aspects of bilateral proximal tibia.

MRI of the right knee revealed low signal intensity fracture line in the medial tibial condyle on T1WI and T2WI. The fracture line was surrounded by extensive bone marrow edema appearing hyperintense on STIR and PDFS sequences consistent with stress fracture. As patient had similar pain in the left knee also, MRI of the other knee was also done for academic purpose. Both the knees showed similar findings on MRI sequences [Fig.1,2] confirming the diagnosis of synchronous 
symmetrical stress fractures of bilateral medial tibial condyles.

Complementary CT [Fig.3] and X-ray [Fig.4] of the both knees revealed horizontally oriented sclerotic lines parallel to the medial growth plates in keeping with synchronous stress fractures of both proximal tibiae.

\section{Discussion}

Stress fractures are usually unilateral, however our patient presented with bilateral stress fractures. Synchronous stress fractures are very rare [2]. A study by McCormick F et al. showed the incidence of bilateral injury in a patient presenting with a stress fracture was $16 \%$ [3]. Our case was a military man who presented with stress fractures after intense physical activity. Hence, stress fractures occur in a bone due to a mismatch of bone strength and chronic mechanical stress placed upon the bone. In India, two studies by Agrawal PK and Dash N et al., have reported high incidence of stress fractures of $11.4 \%$ and $7.04 \%$ in two different military training centers $[4,5]$.

The activities involved in the diverse types of military training may put personnel at different injury risks. The most frequently reported cause of these fractures is repetitive weight-bearing activities such as running and marching, a recent increase in physical activity, beginning of a new activity or some other change in their routine can also result in increase of these fractures [6]. Plain radiographs have poor sensitivity (15-35\%) in early-stage injuries, which increases in latestage injuries (30-70\%). MRI is the modality of choice with a sensitivity reported to reach close to $100 \%$ [7].

The treatment of bilateral stress fractures of the proximal tibia is the same as unilateral stress fractures of the tibia. It consists of rest and protected weight bearing of the limbs, followed by modified activity and the graded return to a training schedule commensurate with bone healing.

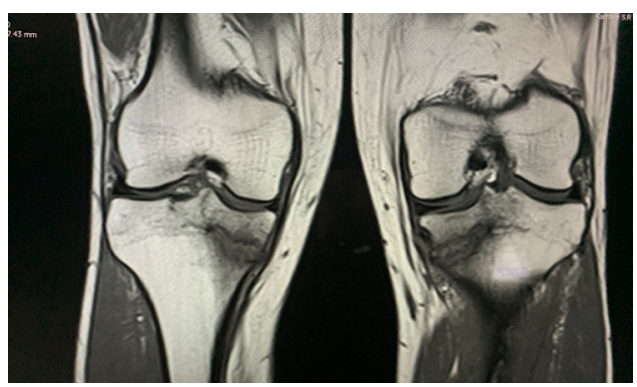

Fig.1: Coronal T1 weighted MRI sequence showing symmetrical low signal intensity fracture lines in the bilateral medial tibial condyles.

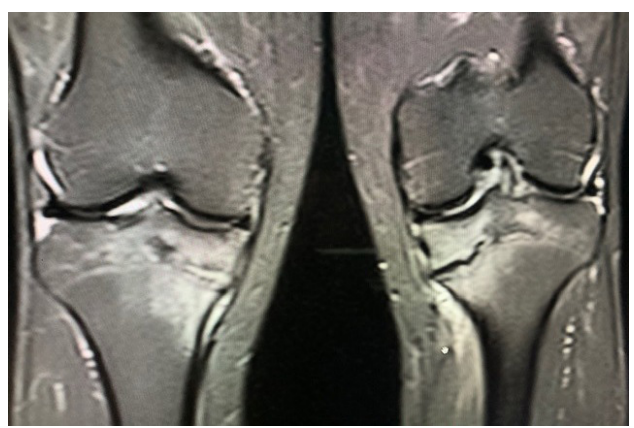

Fig.2: Coronal STIR MRI sequence showing fracture lines surrounded by high signal intensity marrow edema in bilateral medial tibial condyles.

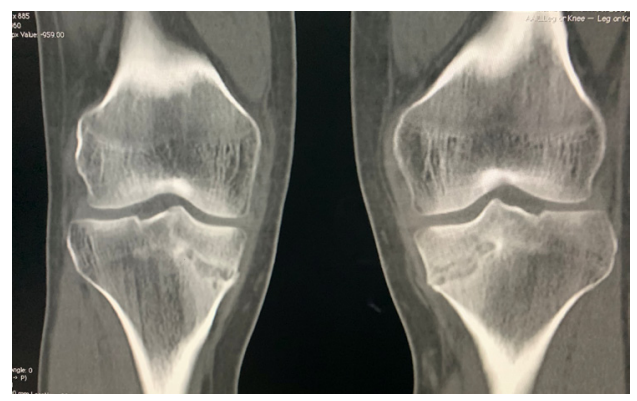

Fig.3: Coronal CT showing symmetrical fractures lines in bilateral medial tibial condyles extending upto the articular surface.

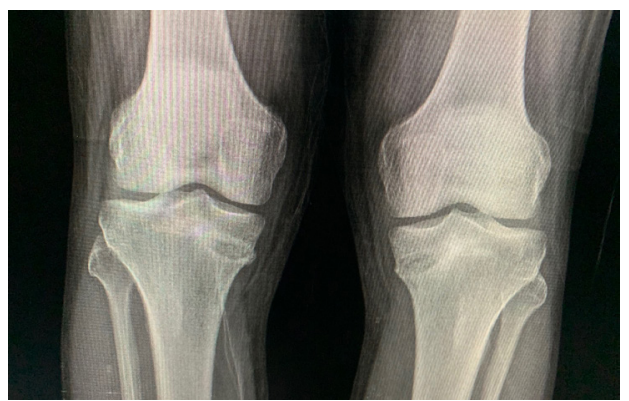

Fig.4: X-ray of bilateral knees showing fracture lines along the medial tibial epiphyseal scars. 


\section{Conclusion}

In the case presented, bilateral proximal medial epiphyseal tibia stress fractures were noted on X-ray, CT and MRI. Hence, in a case of unilateral stress fracture, contralateral side should also be assessed to diagnose bilateral injury as this could help guide treatment decisions as well as inform the patient of the need for lifestyle modifications and continued rest to prevent further injury.

Contributors: MK: manuscript writing, imaging and diagnosis; NKG manuscript editing and imaging. MK will act as guarantor. Both authors approved the final version of this manuscript.

Funding: None; Competing interests: None stated.

\section{References}

1. Boden BP, Osbahr DC, Jimenez C. Low-risk stress fractures. Am J Sports Med. 2001;29:100-111.
2. de Villiers R., Scheepers S. Imaging of Triathlon injuries. In: Guermazi A., Roemer F, Crema M (eds). Imaging in Sports-Specific Musculoskeletal Injuries. Springer, Cham pp. 557-584.

3. McCormick F, Nwachukwu BU, Provencher MT. Stress fractures in runners. Clin Sports Med. 2012;31:291-306.

4. Agarwal PK. Stress fractures-management using a new classification. Indian J Orthop. 2004;38:118-120.

5. Dash N, Kushwaha AS. Stress fractures a prospective study amongst recruits. Med J Arm Forces India. 2012;68:118-122.

6. Jones BH, Thacker SB, Gilchrist J, Kimsey CD Jr, Sosin DM. Prevention of lower extremity stress fractures in athletes and soldiers: a systematic review. Epidemiol Rev. 2002;24(2):228-247.

7. Marshall RA, Mandell JC, Weaver MJ, Ferrone M, Sodickson A, Khurana B. Imaging features and management of stress, atypical, and pathologic fractures. Radiographics. 2018;38(7):2173-2192. 